\title{
CARACTERIZACIÓN Y MITIGACIÓN DEL FLUJO INTERMITENTE EN SISTEMAS DE RECOLECCIÓN MULTIFÁSICOS BAJO CONDICIONES DINÁMICAS
}

\author{
Manuel Cabarcas Simancas ${ }^{1 *}$, Robert Eduardo Padrón García², Gustavo Andrés Valle Tamayo ${ }^{3}$
}

*A quien debe dirigirse la correspondencia

\begin{abstract}
RESUMEN
Los desafíos asociados al flujo multifásico son tan antiguos como la industria del petróleo. En muchos campos de crudo y gas con líneas de flujo multifásico, la inestabilidad de la producción debido a la presencia de flujo intermitente es el principal problema de aseguramiento de flujo. En Colombia, los antecedentes de investigaciones de este tipo son escasos, por consiguiente se plantea la necesidad de realizar estudios similares en nuestro territorio. Existen razones para creer que diferentes campos nacionales presentan problemas de aseguramiento de flujo relacionados con el flujo intermitente. El objetivo de este artículo es realizar una recopilación teórica de los estudios principales relacionados con el flujo intermitente en operaciones de la industria de Petróleo y Gas, que permita sentar las bases para trabajos posteriores en el área, con respecto a esta temática en el ámbito nacional. Este trabajo detalla un estado del arte sobre el flujo intermitente, sus características y una revisión exhaustiva de los métodos de simulación existentes en la literatura. Adicionalmente, plantea una metodología para realizar estudios de este tipo de flujos, al igual que una discusión sobre posibles estrategias de mitigación y su aplicación en campo.
\end{abstract}

Palabras clave: Aseguramiento de Flujo, Flujo Multifásico, Flujo tipo Tapón, Flujo Transiente, Fenómenos de Transporte, Facilidades de Superficie.

\section{CHARACTERIZATION AND MITIGATION OF SLUGGING FLOW IN OIL GATHERING NETWORKS UNDER DYNAMIC CONDITIONS}

\begin{abstract}
Challenges associated to multiphasic flow are as old as the petroleum industry itself. In many oil and gas fields with multiphasic flow lines, production instability due to severe slugging is their main flow assurance issue. In Colombia, the previous works on this matter are rather limited. There are strong reasons to presume the existence of flow assurance issues related to severe slugging in many national fields. The main purpose of this article is to perform a technical compliance of the most relevant studies related to severe slugging in the Oil and Gas Industry operations, which allows to set basis for further works in this area, addressing the matter in the national context. This paper contains a state-of-the-art review on severe slugging, intermittent flow, their characteristics, and a thorough revision of the existing numerical simulation methods in literature. In addition, this document proposes a methodology for the realization of similar studies, and a discussion of mitigation strategies and their application on field operations.
\end{abstract}

Keywords: Flow Assurance, Multiphasic Flow, Slug Flow, Transient Flow, Transportation Phenomena, Surface Facilities.

1. Universidad Industrial de Santander (UIS). Docente Planta. Carrera 27 con Calle 9, Ciudad Universitaria. E-mail: cabarcas@uis.edu.co

2. Universidad Industrial de Santander (UIS). Ingeniero de Producción. E-mail: robert.padron@pccsas.com

3. Universidad Industrial de Santander (UIS). Ingeniero de Producción. E-mail: gustavo.valle@pccsas.com 


\section{INTRODUCCIÓN}

Los desafíos asociados al flujo multifásico son tan antiguos como la industria del petróleo. Sin embargo, a medida que la producción se ha trasladado a ambientes más hostiles, la utilidad del transporte multifásico en las operaciones de producción de crudo y gas ha ganado relevancia (Erickson y Mai, 1999). En muchos campos de crudo y gas con líneas de flujo multifásico, la inestabilidad de la producción debido a la presencia de flujo intermitente es el principal problema de aseguramiento de flujo (Tang y Danielson, 2006). Las tuberías bifásicas están usualmente diseñadas para operar bajo estado estable. No obstante, cuando se inducen cambios en condiciones operacionales tales como tasas de flujo, presiones en el separador; en las tuberías se produce un cambio a estado transiente (Choi et al., 2013). En Colombia, los antecedentes de investigaciones de este tipo son escasos, planteando la necesidad de realizar estudios similares en nuestro territorio. Existen razones para creer que diferentes campos nacionales presentan problemas de aseguramiento de flujo relacionados con el flujo intermitente.

La expresión de flujo multifásico es usada para describir el flujo simultáneo de gas y líquido. Su complejidad radica en la deformidad y compresibilidad de las fases, originando un rango de posibilidades de patrones de flujo (Ghajar, 2005). Su caracterización es crucial para el modelado, diseño y operación de diversos procesos. La problemática en particular radica en los patrones de flujo intermitentes, tales como el flujo tapón, que introducen la inestabilidad y aleatoriedad al sistema (Araujo et al., 2013).

El flujo intermitente generalmente es causado por condiciones operacionales de bajas tasas de líquido y gas, en consecuencia, la capacidad de flujo del líquido en las tuberías puede verse reducida, acumulándose en la base de la inclinación y bloqueando así el paso de gas (Tang y Danielson, 2006). Para una tubería en un terreno escarpado (onshore) o en un sistema tubería-riser en operaciones offshore, a menudo no es posible dar una solución en estado estable, y se desarrollan condiciones para el flujo intermitente (Taitel, 1986). En la literatura se definen tres tipos de tapones: Hidrodinámicos, operacionales y topográficos, contribuyendo a pérdidas de producción de aproximadamente hasta el 68\% (Tang y Danielson, 2006).

La investigación del fenómeno del flujo transiente en la industria del petróleo empezó entre finales de la década de los 70's y principios de los 80 's. Desde ese entonces, la industria ha desarrollado códigos transientes multipropósito tales como OLGA, PLAC, TACITE y más recientemente LEDAFLOW. Estos simuladores normalmente consisten en conjuntos de ecuaciones de conservación de masa, energía y momentum (Choi et al., 2013).

Uno de los primeros estudios del modelado del flujo tapón fue desarrollado por Dukler y Hubbard (1975). Este modelo se basa en una celda unitaria periódica, el cual predice el promedio de las fracciones líquidas y la caída de presión para ciertas tasas de flujo, inclinaciones de tubería y propiedades de fluidos. Una revisión de los modelos de tapones en estado estable se puede encontrar en Taitel y Barnea (1990) y en Fabre y Line (1992).

Bugg et al. (1998) implementaron modelos numéricos en dos dimensiones, basados en diferencias finitas para alongamiento de la burbuja. Taha y Cui (2006) evaluaron el mismo fenómeno en tres dimensiones usando FLUENT, un paquete comercial de CFD.

Durante la década de los 90 's, se realizaron avances relacionados con los modelos de tapones en estado estable, orientados al modelado de tapones hidrodinámicos $\mathrm{y}$ topográficos. Estos utilizaron un enfoque lagrangiano para rastrear las fronteras del tapón a medida que se movilizaba aguas abajo. Los modelos varían desde simples expresiones cinemáticas a modelos complejos, donde el desplazamiento de las fronteras del tapón es contenido en balances de masa y momentum (Rosa et al., 2015).

Inspirado en el comportamiento tipo onda de la velocidad traslacional del tapón, Barnea y Taitel (1993) propusieron un modelo cinemático aplicado a tapones hidrodinámicos para predecir la distribución de la longitud del tapón, basados en el mecanismo de adelantamiento de la burbuja. Zheng et al., (1994) complementaron el modelo anterior con un balance de masa de líquidos, con el fin de rastrear tapones individuales. Cook y Behnia (2000) compararon el modelo de Barnea y Taitel (1993) con resultados experimentales tomados a inclinaciones mayores a $5^{\circ}$.

Nydal y Banerjee (1996) propusieron un algoritmo lagrangiano para el tapón líquido y la burbuja de gas. Los desplazamientos de la interfaz burbuja-tapón son evaluados satisfaciendo los balances de masa y momentum de las fases. Concluyeron en un conjunto de ecuaciones que definen las presiones y las velocidades de los tapones. 
Taitel y Barnea (1998) refuerzan su modelo presentado inicialmente en 1993, con el fin de satisfacer los balances de masa y de momentum. Este modelo de rastreo hidrodinámico desprecia los efectos de adelantamiento de la burbuja y el término de advección, mientras que las ecuaciones de momentum asumen un equilibrio en el balance de fuerzas local para cada celda. Los resultados revelan que los efectos de compresibilidad del gas causan un incremento en la longitud de la burbuja de gas y tiene un efecto menor sobre el crecimiento de la longitud del tapón de líquido aguas abajo.

Al- Safram et al. (2004) extendieron el trabajo realizado por Taitel y Barnea (1998), proponiendo un modelo para aplicaciones en terrenos escarpados, usando el modelo de Zheng et al. (1994) e incluyendo el holdup del tapón de líquido y mecanismos de adelantamiento de flujo. Wang et al. (2006) incluyen este modelo para evidenciar el efecto de la caída de presión debido a la aceleración de la película del líquido, con el objetivo de estudiar la distribución de la longitud del tapón.

Ujang et al. (2006) estudian el tapón líquido y la elongación de la burbuja de gas por separado, para proponer un nuevo modelo hidrodinámico de predicción de tapones. Se incluyen procesos para ajustar la longitud y ocurrencia del tapón en intervalos de tiempos tomados a partir de una distribución exponencial basada en datos experimentales.

Una limitación de los modelos de predicción de tapón consiste en la necesidad de calcular ciertas propiedades mediante correlaciones empíricas (tales como velocidades, longitudes, holdups y frecuencias) en la entrada o en un codo donde el flujo es reiniciado debido a la nueva inclinación de la tubería. Este proceso es llamado iniciación del tapón, en el cual la inestabilidad del flujo evoluciona a un tapón hidrodinámico. Issa y Kempf (2003) demostraron la capacidad de un modelo de dos fluidos para capturar la iniciación del proceso de tapón en flujo horizontal.

Una aproximación alternativa es el desarrollo de un modelo hibrido, a partir de la unión de un modelo de dos fluidos (considerando cada fase por separado) y un modelo de predicción del tapón (tratando ambas fases como una mezcla). Un ejemplo de esto son los modelos planteados por Larsen et al. (1997) y Kjeldy et al. (2013). Muchos autores han propuestos modelos numéricos para determinar la longitud del tapón y su distribución mediante la experimentación (Taitel y Barnea, 1993,1998; Cook, 2000; Nydal, 1996; Wang, 2006).

Varios autores evidencian por medio de casos aplicados las consecuencias del flujo tapón, Alvarez y Al-Awwammi (1999) discuten los resultados de simuladores de flujo en estado estable y transiente utilizados para el diseño, operación y métodos de solución del complejo multifásico de líneas de flujo en un campo en Arabia Saudita. Tang y Danielson (2006) exhiben los problemas del flujo tapón con respecto al nivel de líquido en el separador, cierres operacionales y pérdidas de producción en la facilidad ConocoPhillips Alpine, al norte de Alaska.

Estos autores proponen mecanismos de mitigación, dentro de los cuales se incluyen: (i) cambiar gradualmente la apertura de la válvula de control de la entrada, (ii) reducir el acceso de la válvula de choque del separador, y (iii) Aplicar una válvula de control automática basada en un sistema de control mejorado del nivel de líquido (Tang y Danielson, 2006). Igualmente, uno de los métodos para aliviar un taponamiento severo, es mediante el incremento de la presión del separador (Yocum 1973). Taitel(1986) proporcionó una explicación teórica de como los choques pueden estabilizar el flujo. Se ha demostrado que chocar el flujo puede aliviar el taponamiento severo, con un incremento mínimo en la presión de la tubería (obteniendo la misma tasa de flujo tanto para líquido como para gas). Las válvulas de choque como método correctivo de flujo intermitente, han sido aplicadas exitosamente en repetidas ocasiones, posicionándolas probablemente como el método más práctico para eliminar tapones. (Schmidt et al., 1980).

Para intentar evitar este tipo de flujo intermitente, Alvarez y Al-Alwwami (1999) plantean que las velocidades experimentadas en la tubería pueden o bien disminuirse o aumentarse cambiando el diámetro. Sin embargo hay que tener en cuenta que en tuberías multifásicas, un diámetro mayor podría ser contraproducente (Erikson y Mai, 1999).

El objetivo de este artículo es realizar una recopilación teórica de los estudios principales relacionados con el flujo intermitente en operaciones de la industria de Petróleo y Gas, que permita sentar las bases para trabajos posteriores en el área, con respecto a esta temática en el ámbito nacional. 


\section{CARACTERISTICAS DEL FLUJO INTERMITENTE EN LINEAS DE PRODUCCIÓN}

Un tapón se define como una onda propagada, generada debido al flujo multifásico en una tubería por diversos factores que están relacionados con las propiedades de los fluidos, la hidrodinámica y la geometría de la tubería. Algunos de estos factores son la variación en la velocidad de las fases (hidrodinámica), densidad y viscosidad de los fluidos (propiedades de los fluidos) e inclinación de la tubería (geometría) (Omowunmi et al., 2013).

Un tapón esta descrito tanto por un frente, definido como una onda disruptiva continua, y una cola que se encuentra en perpetua dispersión, seguido por una burbuja de gas. La combinación del tapón de líquido y la burbuja de gas, se define como unidad tapón (Ver Figura 1). Una vez se inicia, el tapón puede crecer o reducirse dependiendo de las velocidades relativas del frente y de la cola (Omowunmi et al., 2013).

La creación de tapones genera oscilaciones en el campo, una carga excesiva en las facilidades de separación y operación, además de aportar al desgaste de los equipos. Posterior a su iniciación, la presión de la burbuja de gas crecerá, forzando eventualmente a que el tapón de líquido se acelere rápidamente hacia arriba, resultando en un surgimiento de flujo de líquido significativamente alto, seguido de un incremento en el flujo de gas. A medida que se presenta esta alta tasa de gas, la presión aguas arriba disminuye. Finalmente, el caudal de gas declina y el líquido se acumula en la base de la inclinación, iniciando así el nuevo ciclo del tapón. (Tang y Danielson, 2006).

El fenómeno de entrampamiento de gas podría impactar en la tasa de crecimiento del tapón, o por el contrario podría estabilizarlo reduciendo la densidad de la fase líquida (Schulkes, 2007).

Existen 3 tipos de Taponamiento (Tang y Danielson, 2006):

- Tapones Hidrodinámicos: estos se forman debido a la inestabilidad de las ondas a ciertas tasas de flujo de gas y líquido.

- Tapones Operacionales: generados por el cambio de las condiciones de flujo, tales como operaciones de marraneo, cierre de operaciones y aumento de tasas de flujo.

- Tapones Topográficos: Causados por la acumulación y la extracción continua de líquido en las inclinaciones de las líneas de flujo a bajas tasas

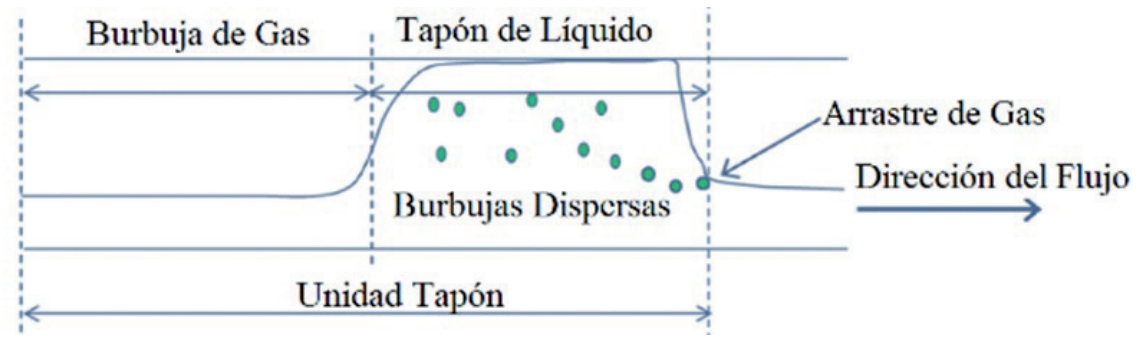

Figura 1. Descripción de Flujo tapón en una tubería horizontal. Adaptado de Omowunmi et al., 2013

El taponamiento inducido por la topografía del terreno, se desarrolla en una sección colina-abajo de una tubería o en la base de un riser, ocasionando que el fluido se acumule en el fondo por acción de la gravedad (Omowunmi et al., 2013). En contraste, los tapones hidrodinámicos se originan a partir de un flujo estratificado debido a perturbaciones en la interfaz gaslíquido. Una onda se propaga y eventualmente crece en la tubería para formar un tapón (Lin, 1986). Los tapones hidrodinámicos son usualmente caracterizados por sus altas frecuencias de irregularidad en comparación a los tapones topográficos (Omowunmi et al., 2013).
Tres factores principales contribuyen a la formación de tapones: (1) cambios en la elevación del terreno, (2) bajas tasas de flujo y (3) sobredimensionamiento de las líneas. La inestabilidad se debe a la combinación de las bajas tasas de flujo, diámetros de tubería sobredimensionados y perfiles topográficos desfavorables. La formación de tapones produce oscilaciones, provocando un inapropiado control de los líquidos recibidos en el separador, y consecuentemente incrementa ndo el desgaste y ruptura de los equipos. Esto puede causar un pobre desempeño operacional, paradas del separador, o altas quemas de gas (Tang y Danielson, 2006; Alvarez y Al-Alwwami, 1999). 


\section{MODELADO DEL FLUJO INTERMITENTE}

Un análisis de estado estable puede proveer una aproximación de primer orden para estimar la capacidad de manejo de líquido bajo diversos escenarios operacionales. Esto es particularmente útil para la evaluación de un número de opciones de desarrollo de un campo mediante estudios paramétricos y para la identificación de situaciones futuras, en las cuales las restricciones de capacidad sean importantes. Sin embargo, se requiere un análisis transiente más riguroso para estimar los requisitos de capacidad de manera precisa y para tener en cuenta prácticas operacionales dependientes del tiempo para el manejo de fluidos. Por ejemplo, el análisis transiente es necesario para cuantificar la reducción en el tamaño de un slug catcher resultante de un aumento de producción gradual y no instantáneo (Ellul et al., 2004).

El modelo OLGA (Bendiksen et al., 1991) es uno de los más empleados en la industria para la simulación de flujo intermitente, basado en una extensión de un modelo dos-fases. OLGA está compuesto de tres ecuaciones de continuidad: una para la fase gaseosa, otra para la fase líquida continua y una para las gotas de líquido. También se encuentran dos ecuaciones de momentum presentes: una combinada para las fases gaseosas y de líquida en gotas, y otra separada para la fase líquida continua. Además de estas, existe una ecuación general de conservación de la energía.

La ecuación de balance de masa para la fase gaseosa se encuentra expresada en la Ecuación 1.

$$
\frac{\partial}{\partial t}\left(f_{G} \rho_{G}\right)=-\frac{1}{A} \frac{\partial}{\partial z}\left[A f_{G} \rho_{G} v_{G}\right]+\psi_{G}+G_{G}
$$

La Ecuación 2 muestra el balance de masa para la fase líquida continua.

$$
\begin{gathered}
\frac{\partial}{\partial t}\left(f_{L} \rho_{L}\right)=-\frac{1}{A} \frac{\partial}{\partial z}\left[A f_{L} \rho_{L} v_{L}\right]-\psi_{G} \frac{f_{L}}{f_{L}+f_{D}}- \\
\psi_{e}+\psi_{d}+G_{L}
\end{gathered}
$$

Por último, la expresión para el balance de masa de la fase líquida en gotas se aprecia en la Ecuación 3:

$$
\begin{gathered}
\frac{\partial}{\partial t}\left(f_{D} \rho_{D}\right)=-\frac{1}{A} \frac{\partial}{\partial z}\left[A f_{D} \rho_{L} v_{D}\right]-\psi_{G} \frac{f_{D}}{f_{L}+f_{D}}+ \\
\psi_{e}-\psi_{d}+G_{D}
\end{gathered}
$$

Donde $f_{G}, f_{L}, f_{D}$ simbolizan las fracciones volumétricas de las fases gaseosas, líquidas continuas y líquida en gotas respectivamente, mientras que ' $\rho$ ', ' $v$ ' $y$ ' $p$ ' representan la densidad, velocidad y presión, y 'A' constituye el área transversal de la tubería. Los subíndices ' $G$ ', 'L', 'i' y ' $D$ ' indican gas, líquido (continuo), interfaz y líquido (gotas) correspondientemente. La tasa de transferencia de masa entre las fases es representada por $\psi_{G}$, mientras que $\psi_{e}$ y $\psi_{d}$ hacen referencia a las tasas de encrustamiento y acumulación. Por último ' $G$ ' representa una fuente de masa, de la fase respectiva a su subíndice.

La ecuación para el balance de momentum combinado para la fase Gaseosa y líquida en forma de gotas se puede expresar como lo muestra la Ecuación 4:

$$
\begin{gathered}
\frac{\partial}{\partial t}\left(f_{G} \rho_{G} v_{G}+f_{D} \rho_{L} v_{D}\right)=-\left(f_{G}+f_{D}\right)\left(\frac{\partial p}{\partial z}\right) \\
-\frac{1}{A} \frac{\partial}{\partial z}\left[A f_{G} \rho_{G} v_{G}{ }^{2}+A f_{D} \rho_{L} v_{D}{ }^{2}\right]-\lambda_{G} \frac{1}{2} \rho_{G} v_{G}\left|v_{G}\right| \frac{S_{G}}{4 A} \\
-\lambda_{i} \frac{1}{2} \rho_{G} v_{R}\left|v_{R}\right| \frac{S_{i}}{4 A}+\left[f_{G} \rho_{G}+f_{D} \rho_{L}\right] g \cos a+\psi_{G} \\
\frac{f_{L}}{f_{L}+f_{D}} v_{a}+\psi_{e} v_{i}-\psi_{d} v_{D}
\end{gathered}
$$

Por su parte, la expresión para el balance de momentum de la fase líquida es la observada en la Ecuación 5:

$$
\begin{gathered}
\frac{\partial}{\partial t}\left(f_{L} \rho_{L} v_{L}\right)=-\left(f_{L}\right)\left(\frac{\partial p}{\partial z}\right)-\frac{1}{A} \frac{\partial}{\partial z}\left[A f_{L} \rho_{L} v_{L}^{2}\right] \\
-\lambda_{L} \frac{1}{2} \rho_{L} v_{L}\left|v_{L}\right| \frac{S_{L}}{4 A}+\lambda_{i} \frac{1}{2} \rho_{G} v_{R}\left|v_{R}\right| \frac{S_{i}}{4 A} \\
+f_{L} \rho_{L} g \cos a-\psi_{G} \frac{f_{L}}{f_{L}+f_{D}} v_{a}-f_{L} d\left(\rho_{L}-\rho_{G}\right) g \\
\frac{\partial f_{L}}{\partial z} \operatorname{sen} \alpha-\psi_{e} v_{i}+\psi_{d} v_{D}
\end{gathered}
$$

Donde $\alpha$ representa la inclinación de la tubería, $S_{G}, S_{L}$ y $S_{i}$ son los perímetros mojados para el gas, el líquido y la interface respectivamente. La velocidad, $\mathrm{v}_{\mathrm{a}}$, es igual a la velocidad del líquido, gas o de las gotas respectivamente, dependiendo si ocurre evaporación o condensación. La velocidad relativa, $\mathrm{v}_{\mathrm{g}}$, está definida por una fórmula de distribución de deslizamiento dada en Bendiksen et al., 1991. La velocidad de la interfaz, $\mathrm{v}_{\mathrm{i}}$, se aproxima a $\mathrm{v}_{\mathrm{L}}$.

Por último la ecuación final de conservación de la energía queda expresada en la Ecuación 6: 


$$
\begin{gathered}
\frac{\partial}{\partial t} m_{G}\left(E_{G}+\frac{1}{2} v_{G}^{2}+g h\right)+m_{L}\left(E_{L}+\frac{1}{2} v_{L}^{2}+g h\right) \\
\left.+m_{D}\left(E_{D}+\frac{1}{2} v_{D}^{2}+g h\right)\right] \\
=-\frac{\partial}{\partial z}\left[m_{G} v_{G}\left(H_{G}+\frac{1}{2} v_{G}{ }^{2}+g h\right)\right. \\
+m_{L} v_{L}\left(H_{L}+\frac{1}{2} v_{L}^{2}+g h\right) \\
+m_{D} v_{D}\left(H_{D}+\frac{1}{2} v_{D}^{2}+g h\right)+H_{S}+Q
\end{gathered}
$$

Donde ' $E$ ' representa la energía interna por unidad de masa; 'H' es la entalpía; ' $h$ ' indica la elevación; 'Hs' hace referencia a la entalpía de las fuentes másicas y 'Q' es el calor transmitido por la tubería. Existen ecuaciones auxiliares que representan la presión y la transferencia de masa entre interfaces mediante series de Taylor, el cual puede ser encontrado en Bendiksen et al., 1991 o en Ellul et al., 2004.

La técnica de detección de tapones del OLGA emplea una aproximación unificada y no requiere ninguna correlación del usuario para calcular el hold-up de líquido. Sin embargo, depende de correlaciones empíricas para definir la frecuencia del tapón (Omowunmi et al., 2013). El modelo de control de tapones (OLGA) atribuye un nuevo tapón en una sección si se cumplen ciertos criterios de control. Estos criterios son: (i) Cuando el régimen de flujo de una sección de frontera cambia de flujo separado a tapón, (ii) cuando se requiere generar otro frente de tapón a una cierta distancia y (iii) cuando el tiempo transcurrido desde el anterior tapón sea mayor que un tiempo mínimo específico. El esquema del modelo de control de tapones determina cuando los tapones se crean o se dispersan. Se requiere entonces una frecuencia de tapón inicial o un tiempo de espera entre la formación de los tapones. El tiempo de espera es usado para retrasar el inicio de un nuevo tapón, cuando este ya ha sido formado en un paso de tiempo anterior (Tang y Danielson, 2006).

El modelo de detección de tapones de OLGA usa una constante de retraso (DC, delay constant) para determinar el tiempo requerido entre la generación de tapones en una sección en particular. El tiempo de retraso entre nuevos tapones se determina mediante la Ecuación 7.

$$
\Delta t=D C \frac{d}{V_{L}}
$$

En donde, 'd' representa el diámetro de la tubería en metros, ' $V_{L}$ ' es la velocidad real del líquido en metros por segundo, ' $\Delta \mathrm{t}$ ' es el tiempo de retraso en segundos $\mathrm{y}$ 'DC' representa la constante de retraso.
Nótese que la constante de retraso puede ser definida en base a la velocidad real del líquido en vez de su velocidad superficial. El tiempo de retraso está inversamente relacionado con la frecuencia de aparición del tapón, es decir, la ecuación 7 puede ser reorganizada en la Ecuación 8.

$$
D C=\frac{V_{L}}{d \cdot F_{S}}
$$

Donde, 'Fs' = frecuencia del tapón, tapón/segundo.

En la simulación OLGA, se suele utilizar por defecto un valor de 150 para la constante de retraso. Shea et al. (2004) sugirieron el uso de una correlación empírica (Ecuación 9) para corroborar la frecuencia predicha por OLGA, con el fin de asegurar que esta se encuentre en un rango razonable.

$$
F_{S}=\frac{0.47 V_{S L}^{0.75}}{d^{1.2} L^{0.55}}
$$

Donde ' $\mathrm{V}_{\mathrm{SL}}$ ' representa la velocidad superficial promedio del líquido (en metros por segundo) y ' $L$ ' indica la longitud de la tubería en metros.

En cuanto a herramientas software para simulación de flujo transiente, existen diversas opciones comerciales, basadas en los enfoques planteados anteriormente. Muchas de estas han sido probadas con modelos reales de campo, otorgando ajustes satisfactorios (Danielson et al., 2012; Kjolaas et al., 2013). De igual manera, han sido comparados con datos de laboratorio, especialmente OLGA y LEDAFLOW. La diferencia de resultados, en cuanto a características del tapón y caídas de presión, son despreciables (Belt et al., 2011). La Tabla 1 muestra un resumen de las herramientas comerciales disponibles en la actualidad. Para que la simulación del flujo intermitente sea exitosa, se debe garantizar que los parámetros de entrada sean añadidos con rigurosidad. Dentro de estos parámetros, se encuentran las propiedades de los fluidos, los perfiles topográficos (al igual que la geometría de las líneas, con el propósito de contemplar la formación de tapones inducidos topográficamente en el modelo), los indicadores de producción del campo (con el fin de realizar el ajuste histórico) y la frecuencia con la que se presenta el tapón (como se mencionó anteriormente). A cambio, el software será capaz de caracterizar el tapón adecuadamente, y mediante la simulación se podrán plantear alternativas de mitigación. Este esquema de simulación es ilustrado por la Figura 2. 
Tabla 1. Herramientas comerciales disponibles en la actualidad

\begin{tabular}{|c|c|c|c|}
\hline $\begin{array}{l}\text { HERRAMIENTA } \\
\text { SOFTWARE }\end{array}$ & ESQUEMA DE SIMULACIÓN & PROPUESTO POR & VENTAJAS \\
\hline OLGA & $\begin{array}{l}\text { Modelo Drift de dos fluidos con } 6 \\
\text { ecuaciones de conservación. Realiza } \\
\text { una detección de tapón mediante } \\
\text { estructuras lagrangianas adaptativas. }\end{array}$ & $\begin{array}{l}\text { Bendiksen et al., } \\
\qquad 1991 \\
\text { Nydal and Banerjee } \\
\text { (1996) }\end{array}$ & $\begin{array}{l}\text { Es el software comercial más empleado } \\
\text { para simulación en estado transiente. Los } \\
\text { tiempos de computación son relativamente } \\
\text { bajos con respecto a otras técnicas. }\end{array}$ \\
\hline LEDAFLOW & $\begin{array}{c}\text { Realiza una captura de tapón } \\
\text { mediante múltiples ecuaciones de } \\
\text { fluidos en una malla euleriana. A } \\
\text { diferencia de la detección del tapón, } \\
\text { la geometría del flujo es resuelta en } \\
\text { cada celda. }\end{array}$ & $\begin{array}{l}\text { Issa et al., (1998, } \\
\text { 2003, 2006) } \\
\text { Danielson et al., } \\
2012 . \\
\text { Kjolaas et al., } 2012 . \\
\text { Kjolaas and } \\
\text { Johansenn, 2013. }\end{array}$ & $\begin{array}{l}\text { No requiere ajuste con los datos de } \\
\text { producción, ni el uso de correlaciones } \\
\text { empíricas para el cálculo de la } \\
\text { frecuencia del tapón. Permite una mayor } \\
\text { discretización de las líneas de flujo. } \\
\text { Permite identificar con mayor detalle los } \\
\text { regímenes de flujo transicionales. }\end{array}$ \\
\hline PLAC & $\begin{array}{l}\text { Modelo dos-fluidos, similar } \\
\text { al OLGA. } 6 \text { ecuaciones de } \\
\text { conservación, desarrolladas en } \\
\text { lenguaje FORTRAN. Promedia } \\
\text { propiedades de flujo y depende de } \\
\text { correlaciones empíricas. }\end{array}$ & Black et al., 1990 & $\begin{array}{l}\text { Los tiempos computacionales son } \\
\text { menores, además que le permite al usuario } \\
\text { seleccionar el régimen de flujo tratado. } \\
\text { Las ecuaciones de coeficientes de arrastre } \\
\text { pueden ser resueltas de diversas maneras } \\
\text { (como en un modelo de dos-fluídos, por } \\
\text { ejemplo). }\end{array}$ \\
\hline TACITE & $\begin{array}{l}\text { Modelo de dos fluidos con } 4 \\
\text { ecuaciones de conservación. La } \\
\text { información faltante se asume a } \\
\text { partir de una relación directa con } \\
\text { el régimen de flujo. Las ecuaciones } \\
\text { son resueltas mediante métodos } \\
\text { explícitos. }\end{array}$ & Pauchon et al., 1994 & $\begin{array}{c}\text { Resuelve de manera rigurosa los balances } \\
\text { de masa y energía para cada componente } \\
\text { de la mezcla }\end{array}$ \\
\hline PETRA & $\begin{array}{c}\text { Simulador } 1 \mathrm{D} \text { de tres fases } \\
\text { desarrollado mediante una malla } \\
\text { adaptativa y un código euleriano } \\
\text { en } \mathrm{C}++.\end{array}$ & Larsen et al., 1997 & $\begin{array}{l}\text { Permite hacer un seguimiento individual } \\
\text { de los tapones (al contrario de métodos } \\
\text { promedios) y también permite un } \\
\text { seguimiento de las propiedades del tapón } \\
\text { sin que exista difusión numérica. }\end{array}$ \\
\hline
\end{tabular}

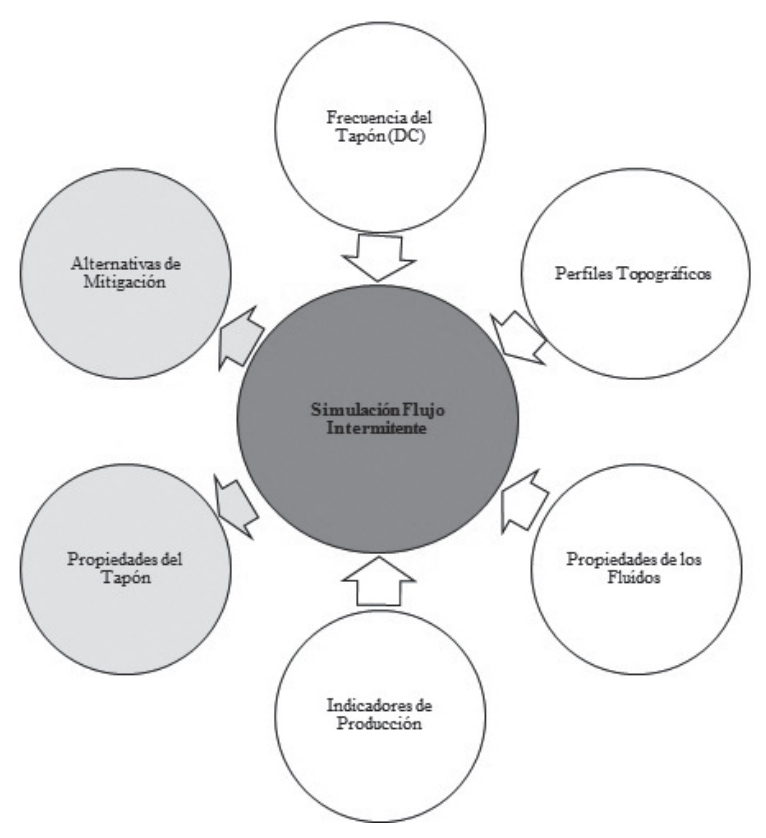

Figura 2. Parámetros de Simulación del Flujo intermitente. 
Los parámetros que caracterizan a un tapón son de particular interés en el modelado de flujo intermitente, ya que permiten hacer el diseño de esfuerzos, además de proveer información importante de su naturaleza física. Estos parámetros son: "Hold-up", Frecuencia, Longitud, Velocidad y Densidad. Es importante aclarar que el análisis de aseguramiento de flujo debe distinguir claramente entre los datos obtenidos para las diferentes secciones del tapón. A menudo se utilizan correlaciones empíricas para caracterizar estos parámetros, sea cual sea el enfoque de simulación. En cuanto a la perspectiva convencional de simulación (dos-fluidos), se resuelve numéricamente un conjunto de ecuaciones simultáneamente para obtener la distribución transiente de las fases a lo largo del dominio de la solución. La técnica de detección de tapones de OLGA depende de correlaciones empíricas para predecir la frecuencia del tapón (Omowunmi et al., 2013).

Una aproximación alternativa es el desarrollo de un modelo hibrido, a partir de la unión de un modelo de dos fluidos (considerando cada fase por separado) y un modelo de predicción del tapón (tratando a ambas fases como una mezcla). Un ejemplo de esto son los modelos planteados por Larsen et al. (1997) y Kjeldy et al. (2013).

El modelo de Larsen et al, sentó las bases para la creación del software LEDAFLOW, caracterizado por tener altos detalles singulares de propiedades de los tapones, pero a expensas de mayores tiempos computacionales. Esta nueva clase de modelos es llamada métodos de captura del tapón. Un gran problema que aún no se ha superado por estos modelos, es tener en cuenta los detalles de mezcla gas-líquido en 3D en un modelo $1 \mathrm{D}$, donde los flujos de entrada son fuertemente influenciados por las condiciones de descarga del mezclador. Asimismo, no hay modelos de captura aplicados a flujo verticales. En adición, esta nueva clase de métodos demanda extensos esfuerzos computacionales. Estos modelos presentan resultados prometedores capturando la iniciación del tapón en líneas horizontales (Rosa et al., 2015).

La Tabla 2 muestra los principales parámetros en la simulación de flujo intermitente, una breve descripción, una correlación empírica y anotaciones sobre su funcionamiento.

Tabla 2. Parámetros Característicos de un Tapón

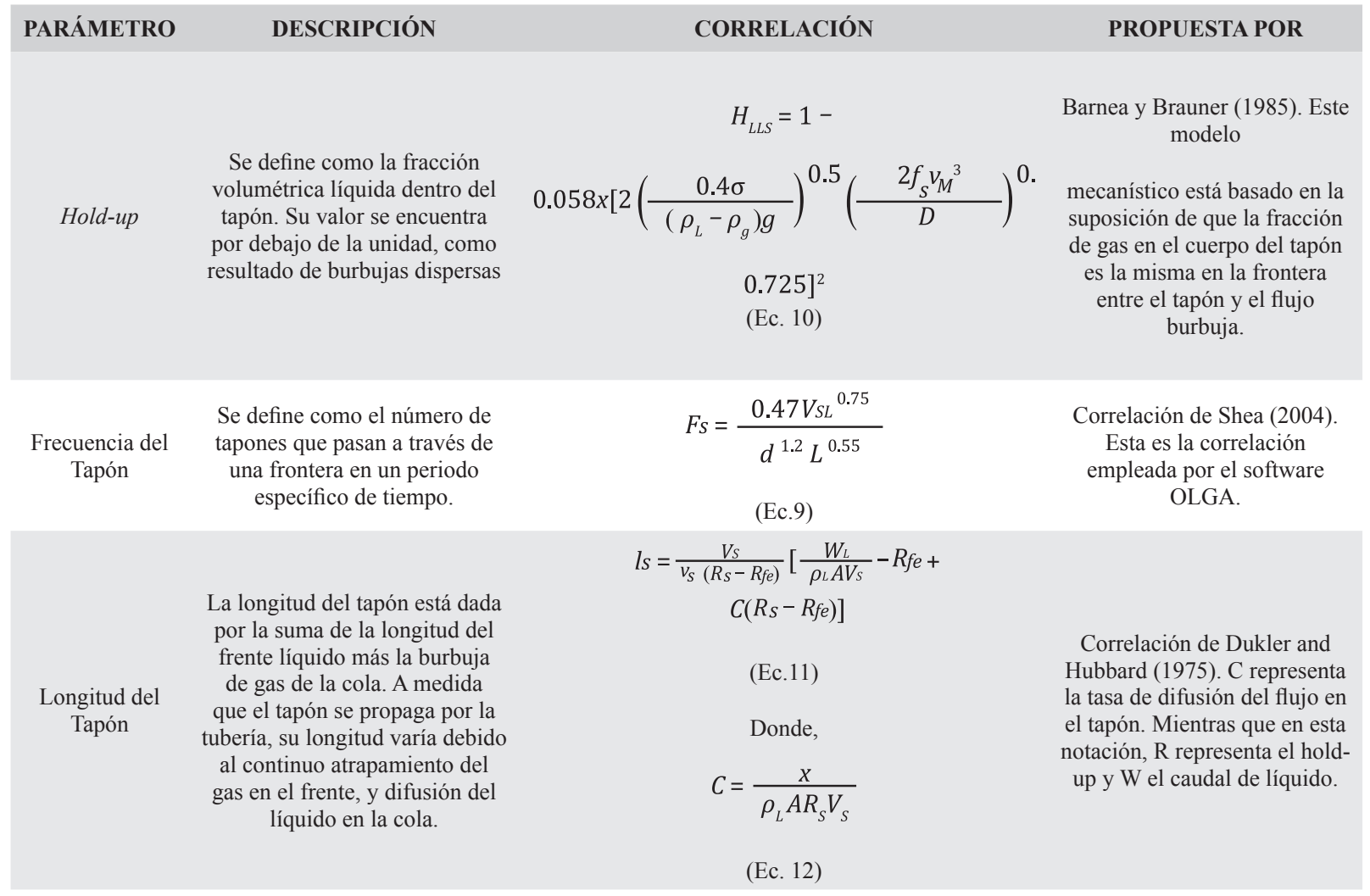




\begin{tabular}{|c|c|c|c|}
\hline PARÁMETRO & DESCRIPCIÓN & CORRELACIÓN & PROPUESTA POR \\
\hline $\begin{array}{l}\text { Velocidad del } \\
\text { Tapón }\end{array}$ & $\begin{array}{c}\text { Un tapón propagado viaja con } \\
\text { una velocidad igual o superior } \\
\text { a la del fluido en estado estable. } \\
\text { La velocidad del tapón es un } \\
\text { parámetro importante para } \\
\text { calcular la fuerza del impacto, } \\
\text { debido a los cambios de } \\
\text { momentum, }\end{array}$ & $\begin{array}{c}U_{S f} \\
U_{G B}\left(1-H_{B S}\right)-U_{C L}\left(1-H_{L S}\right) \\
U_{G B}-U_{G L}\end{array}$ & $\begin{array}{c}\text { Propuesto por Omowunmi } \\
\text { et al., 2013, donde } \mathrm{U}_{\mathrm{GB}} \\
\text { representa la velocidad del gas } \\
\text { en la burbuja y } \mathrm{U}_{\mathrm{GL}} \text { representa } \\
\text { la velocidad del líquido en el } \\
\text { tapón. Los autores mencionan } \\
\text { un valor de } 1.2 \text { a } 1.5 \text { veces } \\
\text { mayor que la velocidad del } \\
\text { líquido }\end{array}$ \\
\hline $\begin{array}{l}\text { Densidad del } \\
\text { Tapón }\end{array}$ & $\begin{array}{l}\text { La densidad del tapón es un } \\
\text { factor fundamental para el } \\
\text { cálculo de esfuerzos, la cual se } \\
\text { deriva a partir de las densidades } \\
\text { de las fases y el hold-up del } \\
\text { tapón. }\end{array}$ & $\begin{array}{c}\bar{\rho}_{L S}=\rho_{L} H_{L S}+\rho_{G}\left(1-H_{L S}\right) \\
(\text { Ec. 14) } \\
\text { Ò, } \\
\bar{\rho}_{B S}=\rho_{L} H_{B S}+\rho_{G}\left(1-H_{B S}\right) \\
\text { (Ec. 15) }\end{array}$ & $\begin{array}{c}\text { Propuesto por Omowunmi et } \\
\text { al., } 2013\end{array}$ \\
\hline
\end{tabular}

\section{METODOLOGÍA PARA DESARROLLAR UN ESTUDIO DEL FLUJO INTERMITENTE}

Un estudio de formación de tapones de líquido necesita determinar variables claves tales como las condiciones para la ocurrencia de tapones, frecuencia, longitud, volumen y estrategias de mitigación de los tapones de líquido (Tang y Danielson, 2006).
La metodología propuesta para el desarrollo del flujo intermitente parte de una disertación de modelos analíticos y simulación numérica (Omowunmi et al., 2013; Tang y Danielson, 2006; Alvarez y Al-Alwwami, 1999), sin embargo en la Figura 3 se presenta el esquema general propuesto en este estudio, el cual será la base para futuras investigaciones aplicadas. Todo el proceso puede ser agrupado en cinco etapas como se relaciona a continuación.

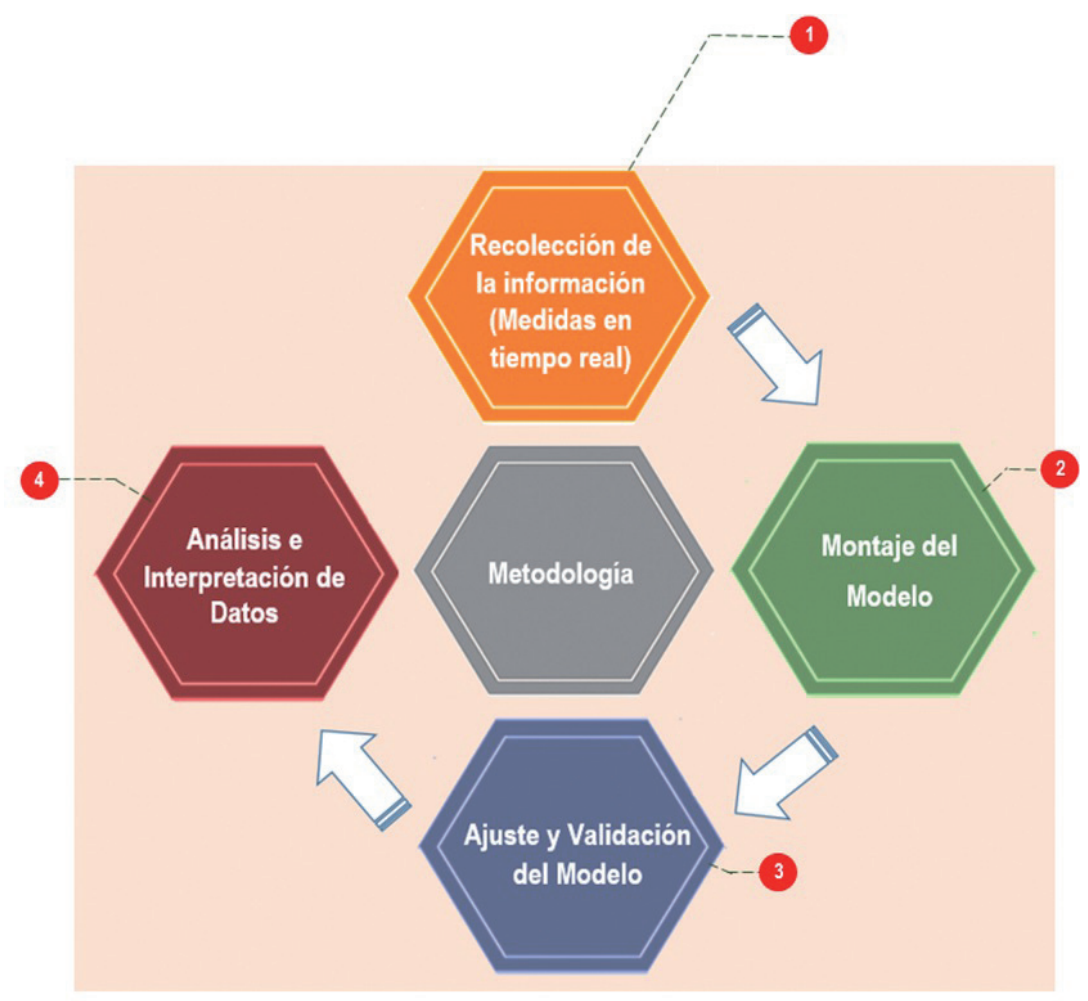

Figura 3. Metodología para desarrollar un estudio de flujo tapón. 
1. Recolección de la Información (Medidas en tiempo real). Composición del fluido, Caudales de fluidos, perfil de presión, gravedad API, gravedad especifica del gas, corte de agua, perfiles topográficos, descripción de las tuberías (ID, material, tipo de aislante, ID del aislante) e instrumentos de la facilidad (válvulas, Controladores, etc.), constante de retraso del tapón. Esta última, será deducida a partir de la Ecu.8., es necesario implementar mediciones de presión dinámica (Presión en tiempo real) para calcular las propiedades del tapón (longitud, frecuencia), se recomienda un periodo de 24 horas para evidenciar de manera correcta el flujo intermitente. Adicional, sino se cuenta con la composición del fluido, esta se determinará mediante la implementación de una prueba ASSAY, que permitirá con ayuda de un software especializado calcularla. Se sugiere realizar un taller diagnostico a modo de preguntas junto con el personal del campo, que confirme flujo intermitente, tales como: ¿Hace cuánto presentan este problema?, ¿La producción de crudo es a bache o constante?, ¿Qué tanto gas produce el campo? ¿Cómo es la apariencia del crudo? ¿Hace cuánto no se realiza una operación de marraneo, son efectivas? Si se confirma la presencia de flujo intermitente, se procede con la metodología.

2. Montaje del modelo. Se procede a construir el modelo en estado estable, con el fin de confirmar el balance de masa y de identificar los patrones de flujo de los colectores, así, se evidenciarán las líneas críticas (flujo intermitente). Seguido, se realiza el montaje en un software de simulación dinámica. Es necesario conocer el PVT del fluido que será ingresado en el simulador. Este podrá obtenerse a partir de:

Opción 1. Composición del fluido. Se ingresa la composición directamente a una herramienta computacional generadora de extensiones PVT, de acuerdo al software en que se modelará el flujo intermitente. Se procede a realizar el montaje del modelo, a través de las características enunciadas en la etapa uno.
Opción 2.ASSAY. Se registra la información de la prueba en un software generador de composiciones. Se sigue el procedimiento enmarcado en la opción 1.

3. Ajuste y Validación de Modelo. Por medio de la constante de retraso del tapón se logra el ajuste buscado, se recomienda empezar con un valor de 150 (Tang y Danielson, 2006). Sin embargo, al ser un valor iterativo, se debe reducir el rango de incertidumbre por medio de una campaña de medición de presión de aquellas zonas críticas identificadas en el simulador en estado estable. Así, se evidenciara la frecuencia y longitud del tapón en los registros de presión. El modelo se valida comparando datos medidos con los calculados por el software (balance de masa y ajuste comportamiento de perfil de presión).

4. Análisis e interpretación de datos. Se plantean escenarios que mitiguen el desarrollo de flujo intermitente.

\section{ESTRATEGIAS DE MITIGACIÓN Y ELIMINACIÓN DEL FLUJO INTERMITENTE}

Dentro de las estrategias de mitigación y/o eliminación de tapones se encuentran las modificaciones en el diseño, que incluye: disminución del diámetro interno de las líneas y el uso de separadores de mayor tamaño o slug catchers; y cambios en la operación del sistema como: implementación de gas lift, estrangulación del flujo (para operar la producción por fuera de la región de formación de tapones) y métodos de control por medio de válvulas. (Tang y Danielson, 2006).

En la Tabla 3 se evidencian algunas estrategias de mitigación empleadas para el flujo intermitente identificadas en la literatura. 
Tabla 3. Estrategias de mitigación y/o eliminación del flujo intermitente consignados en la literatura

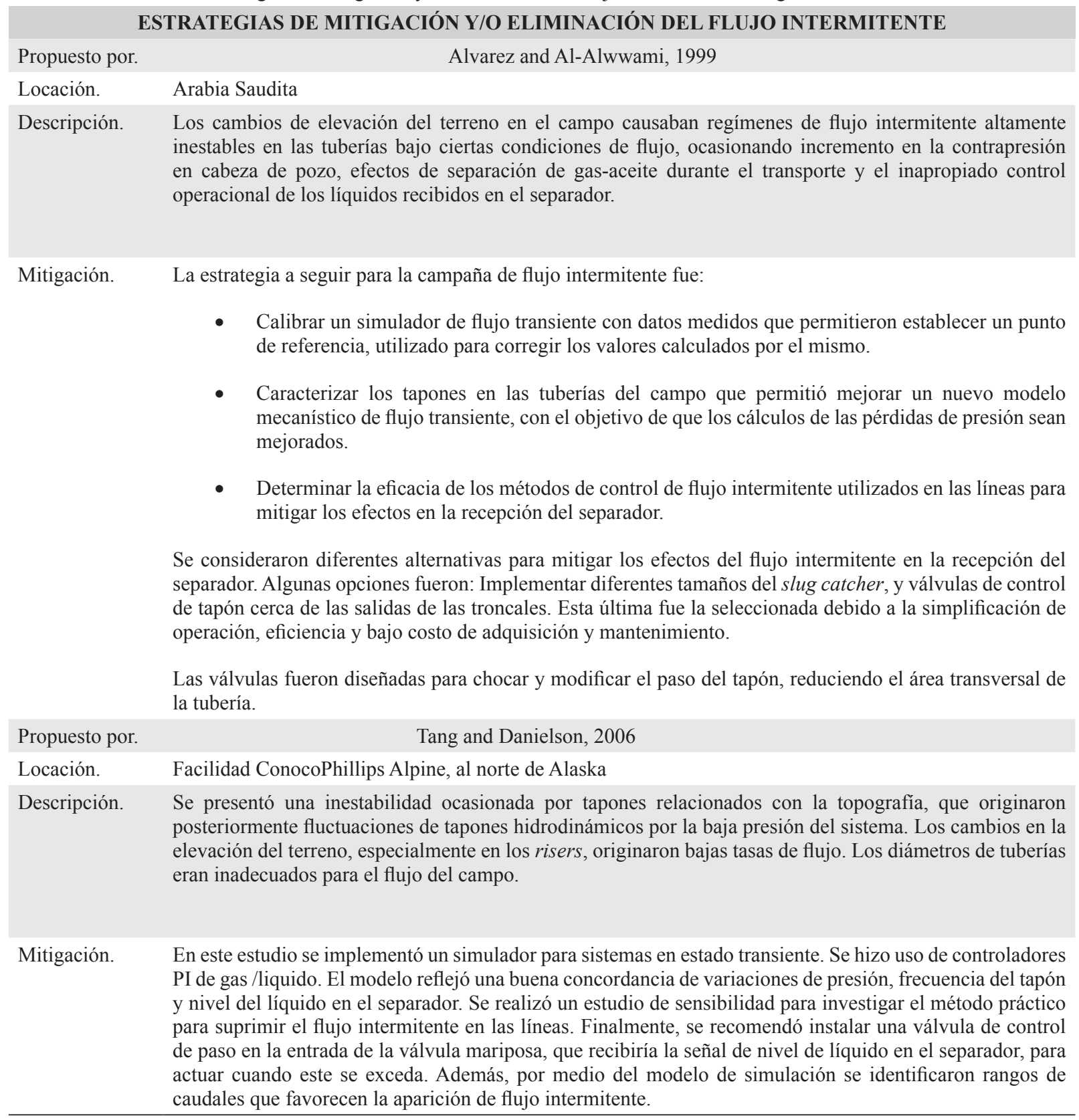

\section{CONCLUSIONES}

- En sistemas de producción multifásicos, es posible que ciertas condiciones desencadenen en un régimen de flujo transiente (tales como cambios operaciones de tasas, bajas velocidades de crudo y gas, además de terrenos topográficamente complejos). El flujo intermitente constituye un problema severo de aseguramiento de flujo en superficie, ya que contribuye con el deterioro del sistema de recolección. Es por esto que su caracterización numérica es importante para la optimización de la producción.

- En la literatura se registran extensivos estudios sobre el modelado numérico del flujo tipo tapón, que han tratado de caracterizar el proceso mediante modelos mecanísticos basados en balances de masa, energía y momentum. Igualmente existen correlaciones empíricas para la caracterización de las propiedades del tapón, tales como la frecuencia, la longitud y el hold-up. Actualmente existe una variedad de herramientas software para el modelado 
del flujo transiente, dentro de las cuales se destaca OLGA.

- En este artículo se propone una metodología para realizar el modelado de flujo intermitente en redes de recolección de crudo en superficie, la cual contempla una caracterización rigurosa del fluido y de las condiciones de operación existentes, el uso de un software comercial para estado transiente y el planteamiento de estrategias de mitigación. Esta metodología ha sido aplicada por los autores en datos de campo en Colombia, obteniendo una caracterización adecuada del flujo tipo tapón en la red, y permitiendo plantear estrategias de mitigación del flujo tipo tapón de manera exitosa y además reportando incrementos en la producción de aceite en aproximadamente un $10 \%$.

Existen diferentes estrategias de mitigación planteadas en la literatura, tales como el uso de válvulas de choque, sistemas de control de nivel e inclusive implementación de sistemas gas lift.

\section{AGRADECIMIENTOS}

Los Autores de este artículo expresan sus más sinceros agradecimientos a Dios y a la Universidad Industrial de Santander por la publicación de esta investigación

\section{REFERENCIAS}

1. AL-SAFRAM EM, TAITEL Y, BRILL JP (2004) Prediction of slug length distribution along a hilly terrain pipeline using slug tracking model. J Energy Resour Technol 126:54-62

2. ALVAREZ, C. J., \& AL-AWWAMI, M. H. (1999, January). SPE 56463. Wet crude transport through a complex hilly terrain pipeline network. In SPE Annual Technical Conference and Exhibition. Society of Petroleum Engineers.

3. ARAÚJO, J. D. P., MIRANDA, J. M., \& CAMPOS, J. B. L. M. (2013). Simulation of slug flow systems under laminar regime: Hydrodynamics with individual and a pair of consecutive Taylor bubbles. Journal of Petroleum Science and Engineering, 111, 1-14.

4. BARNEA D, BRAUNER N (1985) Holdup of the liquid slug in two phase intermittent flow. Int $\mathrm{J}$ Multiph Flow 11:43-49
5. BARNEA D, TAITEL Y (1993) A model for slug length distribution in a gas-liquid slug flow. Int $\mathbf{J}$ Multiph Flow 19(5):829-838.

6. BELT, R., DURET, E., LARREY, D., DJORIC, B., \& KALALI, S. (2011, June). Comparison of commercial multiphase flow simulators with experimental and field databases. In 15th International Conference on Multiphase Production Technology. BHR Group.

7. BENDIKSEN, K. H., MAINES, D., MOE, R., \& NULAND, S. (1991). The dynamic two-fluid model OLGA: Theory and application. SPE production engineering, 6(02), 171-180.

8. BLACK, P. S., DANIELS, L. C., HOYLE, N. C., \& JEPSON, W. P. (1990). Studying transient multiphase flow using the pipeline analysis code (PLAC). Journal of Energy Resources Technology, 112(1), 25-29.

9. BUGG, J. D., MACK, K., \& REZKALLAH, K. S. (1998). A numerical model of Taylor bubbles rising through stagnant liquids in vertical tubes. International journal of multiphase flow, 24(2), 271281.

10. CHOI, J., PEREYRA, E., SARICA, C., LEE, H., JANG, I. S., \& KANG, J. (2013). Development of a fast transient simulator for gas-liquid two-phase flow in pipes. Journal of Petroleum Science and Engineering, 102, 27-35.

11. COOK M, BEHNIA M (2000) Slug length prediction in near horizontal gas liquid intermittent flow. Chem Eng Sci 55:2009-2018"

12. DANIELSON, T. J., BANSAL, K. M., DJORIC, B., LARREY, D., JOHANSEN, S. T., DE LEEBEECK, A., \& KJOLAAS, J. (2012, April). Simulation of slug flow in oil and gas pipelines using a new transient simulator. In Offshore Technology Conference. Offshore Technology Conference.

13. DUKLER AE, HUBBARD MGA (1975) A model for liquid slug flow in horizontal and near horizontal tubes. Ind Eng Chem Fundamentals. 14:337-347"

14. ELLUL, I. R., SAETHER, G., \& SHIPPEN, M. E. (2004, January). The Modeling of Multiphase 
Systems under Steady-State and Transient Conditions-A Tutorial. In PSIG Annual Meeting. Pipeline Simulation Interest Group.

15. ERICKSON,D.D., \&MAI, M.C.(1999).Application of transient-multiphase-flow technology. Journal of petroleum technology, 51(04), 84-87.

16. FABRE J, LINÉ A (1992) Modeling of two-phase slug flow. Annu Rev Fluid Mech 24:21-46

17. GHAJAR, A. J. (2005). Non-boiling heat transfer in gas-liquid flow in pipes: a tutorial. Journal of the Brazilian Society of Mechanical Sciences and Engineering, 27(1), 46-73.

18. ISSA RI, KEMPF MHW (2003) Simulation of slug flow in horizontal and nearly horizontal pipes with the two-fluid model. Int J Multiph Flow 29:69-95

19. ISSA, R.I., WOODBURN, P., (1998). Numerical prediction of instabilities and slug formation in horizontal two-phase flows. 3rd International Conference on Multiphase Flow, ICMF98, Lyon, France.

20. ISSA, R.I., BONIZZI, M., BARBEAU, S. (2006), Improved closure models for gas entrainment and interfacial shear for slug flow modeling in horizontal pipes, International Journal of Multiphase Flow 32 1287-1293.

21. KJELDBY TK, HENKES RAWM, NYDAL OJ (2013) Lagrangian slug flow modeling and sensitivity on hydrodynamic slug initiation methods in a severe slugging case. Int J Multiph Flow 53:2339

22. KJOLAAS, J., \& JOHANSEN, S. T. (2014, July). Terrain Slugging in a High-Pressure Pipeline-Riser System-Large Scale Experiments and Predictions With LedaFlow. In 9th North American Conference on Multiphase Technology. BHR Group.

23. KJØLAAS, J., DE LEEBEECK, A., \& JOHANSEN, S. T. (2013, June). Simulation of Hydrodynamic Slug Flow Using the LedaFlow Slug Capturing model. In 16th International Conference on Multiphase Production Technology. BHR Group.
24. LARSEN M, HUSTVEDT E, STRAUME T (1997) PeTra: a novel computer code for simulation of slug flow. SPE annual technical conference and exhibition, San Antonio, Texas, 5-8 October.

25. LIN, P.Y., HANRATTY, T.J., 1986. Prediction of the initiation of slugs with linear stability theory. Int. J. Multiphase Flow 12, 79-98.

26. NYDAL OJ, BANERJEE S (1996) Dynamic slug tracking simulations for gas-liquid flow in pipelines. Chem Eng Commun 141-142:13-39”

27. OMOWUNMI, S. C., ABDULSSALAM, M., JANSSEN, R., \& OTIGBAH, P. (2013, March). Methodology For Characterising Slugs and Operational Mitigation Strategy Using Olga Slug Tracking Module-Egina Deepwater Project. InOffshore Mediterranean Conference and Exhibition. Offshore Mediterranean Conference.

28. PAUCHON, C. L., \& DHULESIA, H. (1994, January). TACITE: A transient tool for multiphase pipeline and well simulation. In SPE Annual Technical Conference and Exhibition. Society of Petroleum Engineering.

29. ROSA, E. S., MAZZA, R. A., MORALES, R. E., RODRIGUES, H. T., \& COZIN, C. (2015). Analysis of slug tracking model for gas-liquid flows in a pipe. Journal of the Brazilian Society of Mechanical Sciences and Engineering, 1-22.

30. R. SCHULKES, Gas entrainment at a propating slug front, Study group 2007.

31. SHEA R. EIDSMOEN H., NORDSVEEN M., "Slug Frequency Prediction Method Comparison", 4th north american conference on multiphase technology, Canadá, 3-4 June 2004

32. TAHA, T., \& CUI, Z. F. (2006). CFD modelling of slug flow inside square capillaries. Chemical Engineering Science, 61(2), 665-675.

33. TAITEL Y, BARNEA D (1990) Two-phase slug flow. In: Hartnett JP, Irvine TF Jr (eds) Advances in heat transfer. Elsevier, 20, pp 83-132. 
34. TAITEL Y, BARNEA D (1998) Effect of gas compressibility on a slug tracking model. Chem Eng Sci 53:2089-2097.

35. TAITEL, Y. (1986). Stability of severe slugging. International journal of multiphase flow, 12(2), 203217

36. D. BARNEA \& Y. TAITEL, "A model for slug length distribution in gas liquid slug flow, Int. J. Multiphase Flow, Int. J. Multiphase flow, 19, 829838

37. TANG, Y., \& DANIELSON, T. J. (2006, January). Pipelines slugging and mitigation: case study for stability and production optimization. In SPE Annual Technical Conference and Exhibition Society of Petroleum Engineers
38. UJANG PM, LAWRENCE CJ, HEWITT GF (2006) Conservative incompressible slug tracking model for gas-liquid flow in a pipe. 5th BHRG North American conference on multiphase technology, Banff, Canada, May 31

39. WANG X, GUO L, ZHANG X (2006) Development of liquid slug length in gas-liquid slug flow along horizontal pipeline: experimental and simulation. Chinese J Chem Eng 14(5):626-63.

40. YOCUM, B. T. (1973). Offshore riser slug flow avoidance: Mathematical model for design and optimization. Paper presented at SPE London Meeting, SPE 4312, London, UK

41. ZHENG G, BRILL JP, TAITEL Y (1994) Slug flow behavior in a hilly terrain pipeline. Int J Multiph Flow 20:63-79.

Recepción: 22 de Septiembre de 2015

Aceptación: 26 de Octubre de 2015 